\title{
Determination of All Stabilizing Fractional-Order PID Controllers that Satisfy a Weighted Sensitivity Constraint
}

\author{
Yung K. Lee \\ Faculty: Dr. John M. Watkins \\ Department of Electrical Engineering and Computer Science, College of Engineering
}

\begin{abstract}
This paper presents a method for finding all fractional-order (FO) proportional-integral-derivative (PID) controllers that stabilize a given system and at the same time satisfy an $H_{\infty}$ weighted-sensitivity constraint. All the values of the gain parameters of such FO PID controllers are determined in the frequency domain and are given in terms of the proportional gain $K_{p}$, integral gain $K_{i}$, and derivative gain $K_{d}$. In this paper, they were be calculated and plotted on the $\left(K_{p}, K_{i}\right)$ plane with a fixed value of $K_{d}$. This approach can be used even when the transfer function of a system is not available, so long as the frequency response thereof can be obtained.
\end{abstract}

\section{Introduction}

Over the past couple of decades, fractional calculus has gained significant attention as one of the topics that can be applied to a variety of fields in engineering [1]. While PID controllers predominate in the process control field, their non-integer counterpart, or $\mathrm{PI}^{\lambda} \mathrm{D}^{\mu}$ controllers (where $\lambda$ and $\mu$ are arbitrary real numbers) are now being given considerable attention owing to more accurate and natural system modeling and flexibility in design. (Hereinafter, conventional PID controllers are referred to as IO PID controllers to distinguish them from fractional-order (FO) PID controllers.)

In controller design, stability is the utmost important requirement for an FO PID controller to meet, as in the case of IO PID controllers. A complete and combined method for finding all the parameters $K_{p}, K_{i}$, and $K_{d}$ of an FO PID controller that stabilizes a given system of arbitrary order was proposed in [2]. In the paper, all the solutions were determined in terms of the $\left(K_{p}, K_{i}\right),\left(K_{p}, K_{d}\right)$, and $\left(K_{i}\right.$, $\left.K_{d}\right)$ planes using frequency response.

Along with stability, robustness is another important part of controller design. In particular, $H_{\infty}$ control is of importance to guarantee performance as well as robustness of a system. A method for finding all IO PID controllers that stabilize a given system and meet an $H_{\infty}$ weighted sensitivity constraint was proposed in [3].

In this paper, a method was presented for finding all the possible values of the gain parameters $K_{p}, K_{i}$ and $K_{d}$ of FO PID controllers that not only stabilize a given system of arbitrary order but also meet an $H_{\infty}$ weighted sensitivity condition. Because of limited space, the results presented in this paper were limited to the $\left(K_{p}, K_{i}\right)$ plane for a fixed $K_{d}$.

\section{FO PID Controller Design with $H_{\infty}$ Sensitivity Constraint}

Consider the closed-loop system shown in Fig. 1:

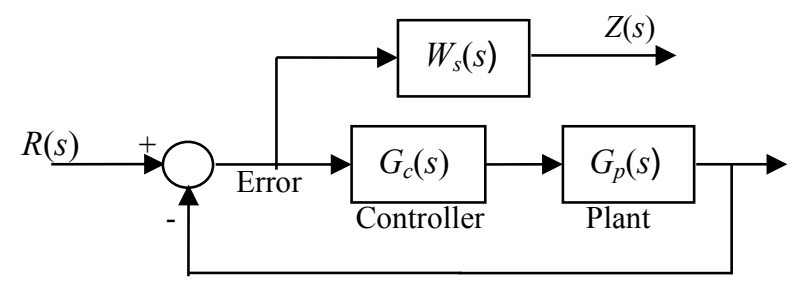

Fig. 1. A closed-loop system with sensitivity function weight

The plant transfer function $G_{p}(s)$ is a single-input single-output (SISO) system, and the transfer function of the $\mathrm{PI}^{\lambda} \mathrm{D}^{\mu}$ (or FO PID) controller $G_{c}(s)$ is given by

$$
G_{c}(s)=K_{p}+\frac{K_{i}}{s^{\lambda}}+K_{d} s^{\mu}
$$

where $K_{p}, K_{i}$ and $K_{d}$ denote the proportional, integral, and derivative gains, respectively, and $\lambda$ and $\mu$ are arbitrary positive real numbers. $W_{s}(s)$ is the sensitivity function weight, $R(s)$ is the exogenous input signal, and $Z(s)$ is the exogenous output signal representing the weighted error. The sensitivity function weight, $W_{s}(s)$, is selected to meet performance specifications as in [4].

\subsection{Solution in $\left(K_{p}, K_{i}\right)$ Plane}

Based on performance requirements, FO PID controllers should meet the following weighted sensitivity constraint:

$$
|S(j \omega)| \leq \frac{\gamma}{\left|W_{s}(j \omega)\right|}, \quad \forall \omega
$$


where $S(j \omega)$ is the frequency domain expression of the sensitivity function $S(s)=\frac{1}{1+G_{p}(s) G_{C}(s)}$ and $\gamma$ is a positive scalar that defines an upper bound of the magnitude of $S(j \omega)$ in conjunction with $W_{s}(j \omega)$.

For a fixed value of $K_{d}, K_{p}$ and $K_{i}$ are given by:

$$
\begin{array}{r}
K_{p}=-K_{d} \omega^{\mu} \frac{\sin \left(\frac{\pi}{2}(\lambda+\mu)\right)}{\sin \left(\frac{\pi}{2} \lambda\right)}-\frac{R_{p}(\omega)-\cot \left(\frac{\pi}{2} \lambda\right) I_{p}(\omega)}{\left|G_{p}(j \omega)\right|^{2}} \\
+\frac{\left(\begin{array}{l}
\cos \left(\frac{\pi}{2} \lambda+\theta_{S}\right)\left(R_{p}(\omega) I_{S}(\omega)-I_{p}(\omega) R_{S}(\omega)\right)+ \\
\sin \left(\frac{\pi}{2} \lambda+\theta_{S}\right)\left(R_{p}(\omega) R_{S}(\omega)+I_{p}(\omega) I_{S}(\omega)\right)
\end{array}\right)}{\gamma \sin \left(\frac{\pi}{2} \lambda\right)\left|G_{p}(j \omega)\right|^{2}} \\
K_{i}=K_{d} \omega^{\lambda+\mu} \frac{\sin \left(\frac{\pi}{2} \mu\right)}{\sin \left(\frac{\pi}{2} \lambda\right)-\frac{\omega^{\lambda} I_{p}(\omega)}{\sin \left(\frac{\pi}{2} \lambda\right)\left|G_{p}(j \omega)\right|^{2}}} \\
-\omega^{\lambda} \frac{\left(\begin{array}{l}
\cos \theta_{S}\left(R_{p}(\omega) I_{S}(\omega)-I_{p}(\omega) R_{S}(\omega)\right)+ \\
\sin \theta_{S}\left(R_{p}(\omega) R_{S}(\omega)+I_{p}(\omega) I_{S}(\omega)\right)
\end{array}\right)}{\gamma \sin \left(\frac{\pi}{2} \lambda\right)\left|G_{p}(j \omega)\right|^{2}}
\end{array}
$$

where

$$
\left|G_{p}(j \omega)\right|^{2}=R_{p}{ }^{2}(\omega)+I_{p}^{2}(\omega)
$$

\subsection{Example}

Consider the following first-order transfer function described in [5]:

$$
G_{p}(s)=\frac{3.13}{433.33 s+1} e^{-50 s}
$$

with a time delay of 50 seconds.

For the performance specifications of a settling time of 1000 seconds, a percent overshoot of $15 \%$, and a steady-state error less than or equal to 0.05 , the method in [4] was used to determine the sensitivity function weight $W_{s}(s)$ :

$$
W_{S}(s)=\frac{0.69224(s+0.007904)}{(s+0.0002736)}
$$

The FO PID controller in [5] with $\lambda=0.8968$ and $\mu=0.4773$ was used to show how effective and useful the method presented here is. Thus, the $\mathrm{PI}^{\lambda} \mathrm{D}^{\mu}$ controller used is given by

$$
G_{c}(s)=K_{p}+\frac{K_{i}}{s^{0.8968}}+K_{d} s^{0.4773}
$$

By using (3) and (4), the weighted sensitivity region and the stability boundary of the FO PID controller were plotted in the $\left(K_{p}, K_{i}\right)$ plane for $K_{d}=4.3867$ and presented in Fig. 2. The weighted sensitivity region represents all the possible values of $K_{p}$ and $K_{i}$ for the fixed $K_{d}$ value of the FO PID controllers (8) that stabilize the plant transfer function (6) and meet the weighted sensitivity condition (2).

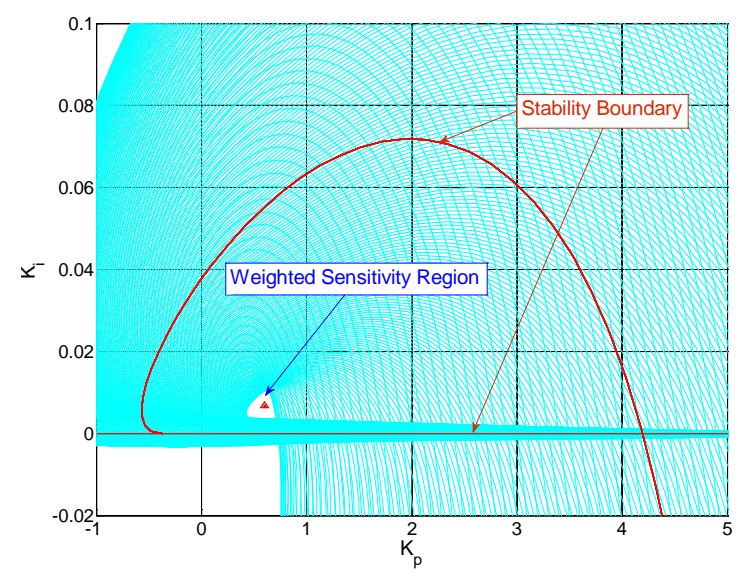

Fig. 2. Stability boundary and weighted sensitivity region in $\left(K_{p}, K_{i}\right)$ plane for the FO PID controller.

\section{Conclusions}

As described in Section 2, a method for finding all the possible values of the gain parameters of $K_{p}$ and $K_{i}$ for a fixed $K_{d}$ value of FO PID controllers that stabilize the plant transfer function (6) and meet the weighted sensitivity condition (2) was presented in this paper.

\section{REFERENCES}

[1] P. R. Hilfer (Ed.), Applications of Fractional Calculus in Physics. Singapore: World Scientific, 2001.

[2] Y. K. Lee and J. M. Watkins, "Determination of all stabilizing fractional-order PID controllers," Proceedings of the 2011 American Control Conference, San Francisco, CA,2011.

[3] T. Emami and J. Watkins, "Weighted Sensitivity Design of PID controllers for Arbitrary-Order Transfer Functions with Time Delay," Proceedings of the IASTED Conference on Intelligent Systems and Control, Orlando, FL, November 2008.

[4] S. Skogestad and I. Postlethwaite, Multivariable Feedback Control Analysis and Design, John Wiley \& Sons, Inc., England, 2005.

[5] C. Monje, Y. Chen, et al., Fractional-order Systems and Controls: Fundamentals and Applications, Springer, London, 2010. 